\title{
Relevance and Barriers to Evidence-Based Practice in Zambian Defense Force Medical Services: A Qualitative Analysis of Roundtable Group Discussion by Military Health Personnel
}

\author{
Omar Abdulwadud ${ }^{1}$, Sailas Nyareza², Floyd Malasha ${ }^{3}$, Justina Phiri Mthoniswa ${ }^{4}$ \\ ${ }^{1}$ Honorary Staff, Cochrane South Africa, Evidence based practice Consultant, Asebe Teferi, Ethiopia. \\ ${ }^{2}$ Graduate Student, Department of Information Science, University of Pretoria, South Africa. \\ ${ }^{3}$ HIV/AIDS Coordinator, HIV Secretariat, Defence Force Medical Services HQ, Ministry of Defence, \\ Lusaka, Zambia. \\ ${ }^{4}$ Program Coordinator, American International Health Alliance, Madison House, MAMCO Building, \\ Independence Avenue, Lusaka, Zambia
}

\begin{abstract}
:
Background: Little is known about the barriers to evidence-based practice (EBP) in military healthcare organization in Africa. We explored the relevance and barriers to EBP among military medical staff working in a Defence Force Medical Services (DFMS) in Zambia.
\end{abstract}

Method: Twenty-three military medical personnel conducted a 90 minutes roundtable discussion using a topic guide. The qualitative data were analyzed by thematic analysis using the OpenCode 4 software.

Results: Three major themes emerged relating to perceived importance of EBP, system and individual-level barriers to EBP. Participants perceived that EBP enhances the cost-effectiveness of treatment, health care quality, patient care and referral system. The system-based barriers were related to lack of administrative support, poor infrastructural, workplace constraints and lack of resources. The individual-based barriers clustered into four subthemes involving shortage of computer skills, motivation, resistance and limited professional development opportunity.

Conclusion: The participants perceived the significance and benefits of EBP in practice contexts. However, they identified 16 system and individual level barriers to effective EBP in DFMS. Recommendations were formulated to overcome the barriers and improve the uptake of EBP.

Keywords: Qualitative study, barriers, evidence-based practice, military medical services, Zambia.

\section{INTRODUCTION}

Evidence-based practice (EBP) is gaining recognition globally as a problem-solving tool to improve a national health system $[1,2]$. EBP stipulates the use of current best evidence with clinical experiences and patient preferences to make sound decisions regarding patient care [3]. During the past two decades, the principle of EBP has been used outside the field of medicine, including nursing [4-8], mental health [9], population health [2], higher education [10] and policy development [11].

Effective implementation of EBP in a healthcare system pays dividends. It enhances healthcare quality $[2,12]$, patient care [13, 14], professional practice [15], decision-making [11] and reduces healthcare cost [9]. Despite these renowned benefits, health systems in developing nations face considerable challenges to apply evidence as a basis for practice. This is supported by evidence from Grol and Grimshaw [16]) who believed that the transfer of evidence to clinical practice and decisionmakings is a complex process and requires meticulous approaches at various levels of care, tailored to specific settings and target groups [16]. Therefore, it is essential to identify the exact barriers and facilitators of EBP at all levels of healthcare system in developing nations.

To-date, many systematic reviews and primary studies have explored the barriers to uptake of EBP in different settings and levels of care outside Africa [2, 4, 5, 8, 11, 13, 15-21]. These studies have 
generated a substantial body of knowledge on EBP barriers. These barriers can be broadly classified as organizational, individual and peer level barriers [20]. In Africa, few such studies have explored the barriers of EBP in public health system and higher education sector [6, 7, 10, 14, 22-29], but none in the military healthcare system. Thus far, evidence is lacking on the relevance and barriers to EBP in military healthcare in Africa. To address this gap in knowledge and provide recommendations, we examined the relevance and barriers to EBP among military medical staffs in Zambian Defense Force Medical Services (DFMS).

\section{Materials ANd Methods}

Study design and setting: During 20-23 August 2013, the American International Health Alliance (AIHA) delivered EBP training for DFMS military medical staff to build their capacity to deliver quality HIV care and treatment services. As a part of the EBP training, military medical staffs conducted a multidisciplinary roundtable group discussion in Lusaka. The purpose of the discussion was to audit and collect baseline information about EBM. The DFMS is a large military health organization serving the Zambian Army, the Zambia Air Force, and the Zambian National Service. It provides healthcare services for both the military and civilian populations. It manages over 50 health facilities consisting of hospitals, clinics, and training institutions across Zambia.

Participants: They were a blend of medical licentiates, clinical officers, and registered nurses and/or midwives, and drawn from various DFMS national military medical institutions, including hospitals, clinics and training schools. The participants knew each other very well and represented different military ranks and geographical locations. All gave verbal consent prior to commencing the discussion. One of the authors (OA) also explained to the participants the purpose of the session, voluntary nature of their participation and guaranteed confidentiality of the information provided.

A clinical officer (CO) is trained in medical model for 3-4 years after secondary education to provide routine care. Medical licentiates (ML) have advanced training in medicine, surgery and offer medical and surgical cares in rural district hospitals. A CO and ML are registered, licensed and the Zambian national regulatory authority [30] regulates their practice. A clinical officer general is licensed to perform general or specialized medical duties such as diagnosis and treatment of disease and injury, order and interpret tests, and perform routine medical procedures. A registered nurse graduates from a nursing school and regulated by a professional council.

Data collection: The 23 participants were assigned to one of the four parallel roundtable discussion groups of five to six per group. The groups elected a moderator, rapporteur, and received a discussion guide in English to explore identical topics for 90 minutes (Box 1). The moderators facilitated, guided and controlled the discussion, and the rapporteurs took notes. Two of the authors (OA, SN) observed the entire discussions and provided support as needed. The handwritten records by rapporteurs were the source of qualitative data.

Box 1: Summary of the topic guide for the multi-disciplinary round table discussion by all four groups (relevance and barriers to evidence-based practice)

Preamble: You have gained some knowledge and skills in EBP from the training workshop. Please discuss the following questions and share your experience.

Topic 1: Relevance of evidence-based practice (EBP)

- Do you think EBP is relevant to your work and your organization?

- How or in what way is it relevant? (Hint: treatment, referral, etc.)

Topic 2: Barriers to evidence-based practice (EBP)

- What are the main barriers (challenges) to implement EBP in your practice or organization? (Hint: support, internet access, resources, training, etc.)

Data analysis: We collected the four handwritten notes, reviewed, typed them as one transcript with group ID, and saved in Microsoft Word as a text file. Two of the authors (OA, SN) read and compared the handwritten notes against the transcript. The data was imported to the OpenCode 4 software [31] and analyzed using thematic analysis. Explicitly, the analysis process involved reading the transcript line by line, creating crude codes, and selective coding and categorization of similar codes to form key themes. We only corrected grammatical errors in the final transcript before analysis but made no substantive changes to the contents. 
Relevance and Barriers to Evidence-Based Practice in Zambian Defense Force Medical Services: A Qualitative Analysis of Roundtable Group Discussion by Military Health Personnel

\section{RESUlTS AND DISCUSSION}

Table 1 shows the participating military medical staffs' demographic details. The participants were full-time employees of DFMS working in rural and urban regions. They represented different specialties, over half were 31-40 years old and 52\% were females. Although English was the first language for only $43 \%$, the majority $(91 \%)$ were very comfortable or comfortable reading and writing in English.

Table 1. Participants demographic details $(n=23)$

\begin{tabular}{|l|l|}
\hline Characteristics & $\mathbf{n}(\%)$ \\
\hline Age groups (years): & \\
30 or less & $3(13.0)$ \\
$31-40$ & $12(52.0)$ \\
$41-50$ & $7(31.0)$ \\
$>50$ & $1(4.0)$ \\
\hline Gender: & \\
Male & $11(48.0)$ \\
Female & $12(52.0)$ \\
\hline Specialty/profession: & \\
Clinical Officer General & $4(17.4)$ \\
Medical licentiate & $2(9.0)$ \\
Clinical Officer & $3(13.0)$ \\
Registered Nurse and /or Midwife & $13(56.3)$ \\
Dental therapist & $1(4.3)$ \\
\hline Work place (practice setting): & \\
Hospital & $4(17.3)$ \\
Clinic & $14(61.0)$ \\
Head office/Administration & $4(17.3)$ \\
Training school & $1(4.3)$ \\
\hline
\end{tabular}

Three major themes emerged from the thematic analysis of the roundtable group discussion data. These themes were "Participants' perceived relevance of evidence-based practice," "Participants' perceived organizational barriers," and "perceived Individual/team barriers" for evidence-based practice at DFMS. The major themes and their particulars are in Table 2 and described as follows.

Table 2. Major themes and subthemes that emerged from group discussion data

\begin{tabular}{|l|l|}
\hline Theme & Subthemes \\
\hline MMSs' perceived benefits of EBP & $\begin{array}{l}\text { Cost-effectiveness of treatment } \\
\text { Enhance healthcare quality } \\
\text { Improve patient care } \\
\text { Improve patient referral system }\end{array}$ \\
\hline MMSs' perceived organizational level barriers & $\begin{array}{l}\text { Administrative constraints } \\
\text { Infrastructural constraints } \\
\text { Lack of resources } \\
\text { Workplace constraints }\end{array}$ \\
\hline MMSs' perceived individual/team level barriers & $\begin{array}{l}\text { Computer illiteracy } \\
\text { Lack of motivation } \\
\text { Lack of professional development } \\
\text { Staff resistance to change }\end{array}$ \\
\hline
\end{tabular}

MMS Military medical staff

Perceived relevance and benefits of EBP: This theme relates to various EBP benefits that participants described, and contains the next four subthemes:

Cost-effectiveness of treatment: The study participants in two of the four groups said the use of EBP in clinical practice will be useful and entails substantial benefits. They affirmed EBP will enhance the 
cost-effectiveness of drugs (interventions) and reduces wastages. The participants in one group said: "EBP is relevant because it reduces empirical treatment and blind therapy. On drugs, it is cost effective and reduces wastage of drugs," (Group 1); whereas the other group mentioned, "Interventions become economical and cost-effective," (Group 4).

Enhancing healthcare quality: Discussants in two groups agreed EBP will have a positive impact on healthcare and would add value to the medical services quality in their organization. They noted: "Evidence based practice promotes quality health services," (Group 1); and the other group stated: "EBP is highly relevant and improves quality of healthcare," (Group 2).

Improving patient care: Discussants in two groups considered the use of EBP in clinical practice would enhance patient care by guiding treatment based on high quality evidence, reducing irrational use of empirical and blind treatment by medical practitioners. They perceived: "EBP is very relevant to us and our organization. It improves patient care," (Group 4); whereas the other group said: "We strongly believe EBP is very relevant to our practice and improves the quality of patient care," (Group 3).

Enhancing patient referral system: Only the participants in one of the four groups agreed that patients' referral is a major problem in their practice settings and need addressing. They believed the use of EBP in clinical practice would have a positive impact on patient referrals by reducing referrals currently taking place. They stated the following: "EBP also reduces unnecessary patient referrals," (Group 4).

Barriers to evidence-based practice: From the participant's roundtable discussion, two barrier themes emerged, that describe the current obstacles to EBP in their DFMS. The barrier themes were: 1) organizational/system-level barriers, and 2) individual/team-level barriers. Table 2 shows the two main themes and their subthemes.

\section{Organizational/system barriers to EBP}

The organizational barrier theme consists of four subthemes of hurdles with potential to influence EBP in participant's organization. These subthemes relate to: 1) Administrative constraints, 2) Infrastructural constraints, 3) Lack of resources, and 4) Workplace (practice setting) constraints. These subthemes are explained below with the participants' quotes.

Administrative constraints: This subtheme describes the lack of support by administrators as well as the inadequacy of leadership at workplace. The participants cited four administrative hurdles at their facilities that would impinge on EBP: "Administrators not attending meetings," "Administrators using internet for private purpose," "Lack of collaboration" and "Lack of guidance for EBP." These four factors are described below:

Participants conversed and agreed their administrators do not attend staff meetings to either communicate about work-related issues. According to them, the lack of good quality managerial support could negatively influence EBP. One group said: "Our administrators are not willing to attend meeting called for by junior ranks and cannot have an effective communication with them," (Group 3). The participants also discussed the use of workplace internet by senior officers. They felt seniors use the workplace internet for personal motive. As a result, they do not get easy access to internet to search for evidence online. Group 3 participants noted: "Occasionally, senior officers are using the internet for other purposes other than learning resource," (Group 3).

Our participants also emphasized the lack of any collaboration with individuals or organizations. They pointed out the current organizational culture does not encourage partnership with individuals or institutions locally in Zambia or overseas. One of the group commented: "Collaboration with local and international institutions and researchers is not that easy, there are so many processes and approvals needed from the Zambian Defense Force senior commanders," (Group 4). The last administrative constraint they discussed was the quality of leadership for EBP. According to the discussants, facility administrators have a major responsibility to support the implementation of EBP, but not doing in their facility. They also confirmed that there is no effective team to lead and facilitate EBP at place of work. One of the participating group commented: "We do not have a working team to guide us and oversee EBP activity at our workplace," (Group 1).

Infrastructural constraints: The study participants highlighted many infrastructural barriers for EBP in the roundtable discussion. Among the infrastructural hurdles they identified are those involving 
Relevance and Barriers to Evidence-Based Practice in Zambian Defense Force Medical Services: A Qualitative Analysis of Roundtable Group Discussion by Military Health Personnel

internet access, learning resource center, library service and a journal club at their workplace. These barriers are described as follows.

There was a consensus among all four groups on the importance of internet access for EBP at a point of care. Overall, the entire groups were unhappy about internet access at workplace and felt it was very poor. They also said they face frequent stoppage from dial in phone connection while searching for evidence online. The discussants reflected: "Internet connection is another major challenge in our facility and it is poor. As a result, it is difficult to have access to relevant online databases at work. For those of us where internet services are available at workplace, there is difficulty in sustaining it because the connection gets interrupted," (Group 1); "The lack of internet connection at workplace prevents us from accessing the online evidence based health information resources like the Cochrane Library. Downloading full-text/PDF articles of systematic reviews or journal an article is often a huge challenge due to intermittent internet connection. Power/electricity availability is also a challenge," (Group 2); "No internet access at workplace. So, we cannot search for research evidence from the online free databases and use them for our practices, " (Group 3); and "Lack of access to internet services to retrieve and use evidence from the available online and free of charge databases for developing countries like the Cochrane Library, Trip database and Pub Med," (Group 4).

The other infrastructural obstacles to emerge from the participants' discussion were a learning resource center (LRC) and library service at workplace. While they agreed that access to a LRC and library service is critical to support knowledge management and build their knowledge base, they were concerned about the lack of these facilities. As a result, they have no sources to access health information. Group 4 members stated: "Lack of learning resources and unavailability of proper libraries at our institutions," (Group 4). Additionally, the lack of a journal club was another barrier emphasized by the participants. Despite acknowledging the essentiality of a journal club in promoting EBP in clinical practice, they expressed the lack of such facility is deterring them from meeting to discuss research articles. They commented: "There is also no journal club at our facility to facilitate and promote EBP and use of research evidence among staff and within the workplace environment" (Group 1); "We do not have a sub journal club to meet and discuss about EBP," (Group 4).

Lack of resources: The participants described that there is insufficient financial and human resources to support EBP in their facilities, especially staff training. They believed that unless the funding and staff level issues are addressed, it is difficult for them to embrace EBP at their facility. One group cited: "Lack of funding to support EBP activities for training is also another major hurdle to advance EBP in our health facilities and in clinical practices," (Group 2); and the other group noted: "No fund available to plan and conduct training workshops for staff," (Group 4). On human resource, the participants in two groups believed that most facilities face staff shortage, and considered this barrier as an impeder for EBP, and make it harder for them to take more tasks when they currently have to cope with a heavy workload. Overtly, participants belonging to two groups said: "There is also heavy work overload and staff shortage at our facility which prevents us to fully and actively engage in EBP activities," (Group 2); and the other group noted: "We also face additional barriers like shortage of manpower," (Group 4).

Workplace constraints: Discussants in two groups envisaged the lack of free time and busyness as their main barriers to engage in EBP at practice setting. They supposed that EBP would impose a further demand on them when they are already on heavy workload and time constraints. They alluded to: "A time factor (constraints) is another obstacle we face. We do not have enough time to read research articles and implement new evidence as we are busy," (Group 1); and the other cluster cited: "There is also heavy workload," (Group 2).

\section{Individual/team related barriers to EBP}

This major theme reflects the personal and peer-related barriers, which participants described as the hurdles for EBP. The theme entails four distinct subthemes involving: 1) computer illiteracy, 2) lack of motivation, 3) poor professional development opportunities, and 4) staff resistance, which are further described as follows.

Computer illiteracy: The study participants perceived that some of their work colleagues lacked basic computer knowledge and skills. Consequently, cannot use workplace computers for EBP and suggested tailored computer training course for computer illiterates. They alleged: "Another obstacle 
to EBP is computer illiteracy among a number of staff in workplaces. Some staffs are not familiar with computers at all and lack the ability to use or work with computers. So, they need to be properly trained to be able to use computers for EBP activities," (Group 2).

Lack of motivation: Participants in one group cited that some of their work colleagues lack appropriate motivation or interest towards EBP. This clearly indicates that such staffs have no shared vision to EBP and require collective support. The participants explained by noting: "There is lack of interest and commitment to EBP by some staff members," (Group 1).

Lack of professional development opportunity: Discussants in one group felt that currently there is no any opportunity for professional development, learn and develop new skills in their organization. Accordingly, they cannot build their knowledge and skills in EBP while working. They highlighted: "Lack of professional development opportunities specifically on developing EBP skills of healthcare professionals is a concern as well," (Group 3).

Staff resistance to change: The discussants in two groups reported that some of their co-workers exhibit negative attitude towards the use of EBP in clinical practice. They added it was hard to engage with them or change their behavior to EBP. They suggested a proper behavioral change intervention to facilitate change in staff attitudes to EBP and its use. They commented: "Behavior change (change of attitude) towards EBP is difficult to bring amid some staff members at work. To address this problem we may need behavior change communication strategy to motive staff to accept and adapt EBP in their daily clinical practice," (Group 2); and "Changing the mindset of co-health workers towards EBP is also very difficult. Perhaps what we need is a behavioral change communication strategy to promote positive attitude and behavior towards EBP among those co-workers resisting to embrace EBP," (Group 3).

This qualitative roundtable discussion study explored the relevance and barriers to EBP in military healthcare organization in Zambia. The study participants have identified 12 system-based and four individual-levels barriers for effective implementation of EBP in the Zambian Defense Force Medical Services (DFMS).

Perceived relevance of EBP: The study participants professed the use of EBP is beneficial in clinical practice, and recognized that it could make treatment cost-effective, improve the quality of health care, patient care, reduce the wastage of drugs and patient referrals. Although there are no comparable studies to judge against our findings, this is consistent with other studies from Africa [6, 7, 10, 14]. A study in Rwanda and Uganda found that over $80 \%$ of hospital doctors and nurses recognized the benefits of EBP and had positive attitude towards research utilization [7]. Thus, for our participants to apply EBP in clinical practice, the principal barriers to EBP they identified in this study should be addressed.

\section{Organizational/system-based barriers to EBP}

The participants identified various system related barriers to EBP that clustered into four groups of constraints. The barriers relate to inadequate administrative support, poor infrastructure, shortage of resources, and those involving workplace.

The participants in this study reported various administrative shortfalls consisting of managers not attending meetings, private use of internet, unhelpful and not guiding. While these barriers are obstructive to EBP and can negatively affect staff morale and motivation. Perhaps, the barriers could be due to communication problems [21] between staffs and managers, administrators' are unaware of their responsibilities or lack EBP knowledge. Findings from a systematic review highlighted that poor institutional support hinders the uptake of EBP [17]. Finding from another study reinforces the critical importance of administrative support by underlining the positive correlation between EBP use and supportive management [6]. Hence, facility administrators must be supportive, lead by example, establish and nurture a culture so that staff may use EBP consistently in their clinical practice.

Medical staffs need essential infrastructure to access evidence and enhance patient care. However, our participants highlighted poor infrastructure as their barrier to EBP. Poor Information and Communication Technology infrastructure and internet access are common problems in Africa [7, 29]. A qualitative study in Senegal found that service delivery providers and community health workers encounter lack of internet access, electrical outages and connectivity difficulty to access, share and use health information in family planning and reproductive health [29]. The majority of 
Relevance and Barriers to Evidence-Based Practice in Zambian Defense Force Medical Services: A Qualitative Analysis of Roundtable Group Discussion by Military Health Personnel

hospital doctors and nurses in Rwanda and Uganda reported poor facilities and internet access to apply EBP [7]. Okwundu [22] also described $80 \%$ of hospital doctors in Nigeria lack access to internet and online databases. Thus, staffs need good infrastructure, reliable internet service and access to online resources to search for evidence to inform practice [21, 32].

The lack of learning resource center, library service and a journal club in a workplace were also the barriers faced by the study participants. The lack of adequate resources and physical facilities for EBP are universal and our finding is consistent with previous research $[7,8,17,21,24,26]$. In a qualitative study in Latin American hospitals, professional informants reported scarce physical facilities as their main hurdle to change perinatal care practice [21]. A library is essential and serves as a source of information for staff to have direct institutional access to online resources, medical journals, and textbooks. Equally, a journal club is important forum for staff to meet and discuss research articles. Therefore, these critical facilities should be provided to facilitate the uptake of EBP in DFMS.

Our participants also cited shortage of staff and funding for EBP activities. Scarcity of resources hinders efficient use of EBP for patient care. Busy staffs under extreme condition may not engage in EBP activities, attend a journal club or search for evidence online [21]. Resource related barriers for EBP however are global predicament [4, 17, 21]. Our finding concur with previous studies [4, 17], which reported that poor resource is a major hurdle to EBP. Hence, it is essential to address the lack of financial and personnel as they can deter staff awareness, receptivity to practice innovations [21], and hold back their efforts to promote EBP in practice.

The final system barrier to EBP participants articulated was a workplace constraint. They stated lack of free time and hectic workload, consistent with other authors from Africa [6, 7, 10, 29] and elsewhere $[4,5,8,17,18]$. These hurdles can deter staff from attending a journal club, searching for articles, reading or integrating research evidence into practice. Perhaps, these barriers could be due to poor work conditions or staff mismatch with work activities. It is imperative to assess and resolve these impediments so that staff participates in EBP activities in their facility. To establish the need for additional staff, a situation analysis of work activities should be conducted to obtain data on staff number, workload, and other vital indicators.

\section{Individual/team-based barriers to EBP}

The participants expressed that there are individual (team) barriers to EBP involving computer skills, motivation, attitude and professional development. They alleged that there are computer illiterate in their workplace that cannot use computers. This finding is consistent with previous studies $[4,17,21]$. A finding from Khammarnia et al study [4] reinforces how common this barrier is. They reported that $69 \%$ of Iranian nurses in a teaching hospital were computer illiterate to implement EBP. Staff competencies and skills influence their interest to new clinical practices, particularly if they lack the skills needed to do new ones [21]. In this age, information technology plays a crucial role in promoting research evidence globally. Health personnel need computer skills to search and access evidence to inform practice. A finding from a previous study reinforce the strong relationship between skills and use of EBP, which highlighted that staff with the right EBP skills are more likely to implement EBP in practice [6].

The study participants stated that there is lack of appropriate motivation towards EBP among some staff. Motivation is a desire or inner force that drives individuals to achieve personal and organizational goals [33]. Staff inspiration is vital for EBP to thrive and influence clinical practice. Motivated staffs can be more productive than those unenthusiastic [33]. The unmotivated staffs need collective support from both peers and administration. Facility managers need to address substandard work conditions and inadequate resources in their facility. They should also recognize that staff personal goals and level of motivation vary. Staff motivation is most critical to effective implementation of EBP. Collective effort is hence vital to encourage staff to contribute to EBP activities within their facilities.

Participants assumed a few of their work-colleagues exhibit a negative attitude to EBP. Negative attitude can negatively affect EBP and common state of affairs in many countries [17, 18, 21, 23]. The possible reasons for pessimistic mind-set include lack of EBP skills, heavy workload, time constraint or other work-related conditions. It is possible to counteract and change a negative attitude through multifaceted professional behavior change interventions tailored to specific settings and target groups 
$[16,19]$. A Cochrane systematic review by Baker et al [15] also found tailored interventions planned to address EBP barriers might change professional practice. In line with these findings, our participants also suggested behavioral change communication strategy to promote positive attitude and behavior towards EBP for associates exhibiting negative attitude.

The participants also expressed concerns about the limited professional development opportunities in their organization. This is a sizeable challenge to implement EBP and may perhaps reflect the lack of staff development policy in DFMS. Staffs need EBP knowledge and skills in order to search evidence-based resources and integrate evidence into clinical practice [35]. Regrettably, health workers in many countries have poor EBP skills [5, 17, 18]. In Africa, the majority of practicing health workers did not learn about EBP at schools. They also have limited prospect to build EBP competency, because there are few institutions in the region that offer a formal EBP course [34]. Consequently, they have no formal EBP training $[6,22]$, skills $[6,24,29]$ and knowledge $[6,10,14$, $22,23,27,28]$ to apply evidence. Competency in EBP is crucial and is recommended for healthcare practitioners [36, 37]. To bridge EBP knowledge and skill barriers, staff require effective EBP education [32]. Thus, DFMS need to explore how best to meet the professional development needs of medical staff in health facilities.

This explanatory study provided insight into the barriers to EBP in the Zambian Defense Force Medical Services (DFMS) that was not available before. The study identified a total of 16 system and individual-based barriers to effective use of EBP. The barriers were mainly around management, leadership, infrastructure, resources, work conditions, computer skills and professional development opportunity, motivation and negative attitude. We recommend the following strategies to overcome these barriers and facilitate the uptake of EBP in DFMS.

\section{A) Implementation strategies for DFMS}

- Establish a centralized EBP committee that can develop the right institutional culture, leadership and support for EBP program throughout DFMS.

- Invest and create a sustainable infrastructure for EBP program in health facilities. In particular, a functional library service, learning resource centre, and dependable internet service to enable staff to access evidence and deliver quality HIV services.

- Assess and allocate adequate resources (financial and human) for EBP program: (a) conduct a situation analysis of existing work arrangements, staff number and workload to validate staff shortages. If established, provide adequate staff to offset staff burnout and heavy workload; (b) revise staff job descriptions and include their roles and responsibilities in EBP program; and (c) allocate adequate fund for EBP program and activities (e.g. training) for health facilities.

- Support staff education, training and skill development using different methods: (a) develop policies and procedures for continuing education/training/professional development opportunities; (b) provide short courses, seminars, workshops, mentoring, fellowship and scholarship opportunities; (c) provide EBP and management trainings for administrators to raise their awareness about EBP, enhance leadership quality and become team players; and (d).encourage facilities and training institutions to initiate EBP seminars, workshops and a journal clubs to promote EBP.

- Enhance staff motivation and attitude to EBP using various strategies: (a) develop and implement a guideline that promotes good personnel management practices; and (b) apply role models, training, enhanced work conditions, and behavioral communication strategy to inspire staff to embrace EBP.

\section{B) Implementation strategies for health facilities}

- That because administrative constraints are obstacles for staff, EBP committee should be established to provide leadership to staff and EBP program at facility level.

- The EBP committee should:

I. Plan, implement, manage, monitor and evaluate EBP program. This includes establishing and maintaining effective communication with staff and senior management in all matters related to EBP program. 
Relevance and Barriers to Evidence-Based Practice in Zambian Defense Force Medical Services: A Qualitative Analysis of Roundtable Group Discussion by Military Health Personnel
II. Address workplace constraints including staff working conditions.
III. Work with senior management to develop suitable infrastructure and adequate resources so that staff can easily access evidence online.
IV. Promote the importance and benefits of EBP among staff by organizing seminars, workshops and a journal club.
V. Undertake an inventory of staff in a facility to identify shortages, maldistribution and allocate adequate staff for EBP program.
VI. Consider enhancing the role of a librarian and learning resource centre coordinator in EBP program.

There are some limitations to this study and the findings require guarded interpretations. Given that we studied 23 military medical staff from a single EBP training, the findings may not be generalizable to all military medical staff or settings in DFMS. Even so, it provides valuable insight into EBP issues that need further exploration. The use of convenience sampling is another limitation and the participants may not be representative. Even so, they came from various facilities, institutions and geographical locations all over Zambia, and symbolized a broad range of specialties, gender and military ranks. The other limitation of this study was that the group discussion was hand recorded and not audiotaped due to the time constraints. Despite the above limitations, our study has strengths. Firstly, we conducted the study as a part of EBP training and with no additional funding. Secondly, it is the first study in a military healthcare organization in Africa and the findings can be used as baseline information to develop tailored interventions to enhance the uptake of EBP in DFMS, and contribute to the evidence-base for EBP in military health services.

\section{Conclusions}

This qualitative study identified several barriers to implementing EBP in Zambian Defense Force Medical Services. We recommend strategies to overcome these barriers and nurture the right institutional culture to support EBP implementation. Our findings would also be a useful benchmark against which future research findings can be compared.

\section{ACKNOWLEDGEMENTS}

The authors wish to thank all military medical personnel from DFMS for their participation in this study. We thank the American International Health Alliance-(AIHA) for funding the development and implementation of the EBP training. The AIHA was supported by PEPFAR and the U.S. Department of Defense through a cooperative agreement between AIHA and the U.S. Department of Human Services, Health Resources and Services Administration. The AIHA is a partner with ZDF since 2007 and has been providing technical assistance to ZDF, including targeted capacity building of medical staff in EBP. Our thanks also to the AIHA Twinning Centre Country Office staff (Zambia) for the logistic support during the EBP training.

\section{REFERENCES}

[1] Committee on Quality of Health Care in America, Institute of Medicine. Crossing the Quality Chasm: A New Health System for the $21^{\text {st }}$ Century. National Academy Press, Washington DC. (2001) [http://nap.edu/10027] ISBN 978-0-309-07280-9 [DOI 10.17226/10027].

[2] Adily A., and Ward JE., Enhancing evidence-based practice in population health: staff views, barriers and strategies for change. Australian Health Review 29(4):469-77 (2005).

[3] Sackett DL., Rosenberg WMC., Gary JAM., Haynes RB and Richardson SS., Evidence-based medicine: what it is and what it is not. BMJ 312: 71-2 (1996).

[4] Khammarnia M., Rezaeian S., Mohammadi MH., Amani Z. and Setoodehzadeh F., Barriers to Implementation of Evidence Based Practice in Zahedan Teaching Hospitals, Iran. Nursing Research \& Practice V 2015 (2014) [http://dx.doi.org/10.1155/2015/357140].

[5] Panagiari D., Barriers and Facilitators for Implementing Evidence-Based Practice among German Nurses working in a General Hospital. Master of Health Sciences Thesis, University of Twente, Enschede-the Netherlands March 2007-Aug 2008 (2008) [http://essay.utwente.n1/59157/1/scriptie_D_Panagiari.pdf]. 
[6] Hadgu G., Almaz S and Tsehay S., Assessment of Nurses' Perceptions and Barriers on Evidence Based Practice in Tikur Anbessa Specialized Hospital Addis Ababa Ethiopia. Am J of Nurs Science 4:73-83 (2015).

[7] Boer K., Barriers to Evidence Based Practice in doctors and nurses in Rwanda and Uganda. Trop Med Int Health;17 Suppl 1:3-80 (2012).

[8] Mehrdad N., Salsali M. and Kazemnejad A., The spectrum of barriers to and facilitators of research utilization in Iranian nursing. J. of Clinical Nursing 17(16):2194-02 (2008).

[9] Fortney JC., Pyne JM, and Burgess JF Jr., Population-level cost-effectiveness of implementing evidence-based practices into routine care. Health Serv Res 49(6):1832-51 (2014).

[10] McInerney P, and Suleman F., Exploring Knowledge, Attitudes, and Barriers Toward the Use of Evidence-Based Practice amongst academic healthcare practitioners in their teaching in a South African University: a pilot study. Worldviews Evid Based Nurs 7:90-7 (2010).

[11] Murthy L, Shepperd S, Clarke M, Garner SE, Lavis JN, Perrier L, et al., Interventions to improve the use of systematic reviews in decision-making by health system managers, policy makers and clinicians. CDSRs Issue 9 Art No CD009401 DOI: 10.1002/14651858.CD009401.pub2. (2012)

[12] Grimshaw J, Eccles M, Thomas R, MacLennan G, Ramsay C, Fraser C and Vale L., Toward Evidence-Based Quality Improvement: Evidence (and its Limitations) of the Effectiveness of Guideline Dissemination \& Implementation Strategies 1966-1998. J Gen Intern Med 21(Suppl 2): S14-S20 (2006)

[13] De Smedt A., Buyl R.., and Nyssen M., Evidence-based practice in primary health care. Studies in Health Technology and Informatics 124:651-6 (2006).

[14] Ntaganira J., Knowledge, attitudes and practice of evidence-based medicine in doctors and nurses in Rwanda and Uganda. Trop Med Int Health 17 Suppl 1:3-80 (2012).

[15] Baker R, Camosso-Stefinovic J, Gillies C, Shaw EJ, Cheater F, Flottorp S, et al., Tailored interventions to address determinants of practice. CDSRs Issue 4. Art. No: CD005470. DOI:10.1002/14651858.CD005470.pub3 9 (2015).

[16] Grol R., and Grimshaw J., From best evidence to best practice: Effective implementation of change in patients' care. The Lancet 362:1225-30 (2003).

[17] Sadeghi-Bazargani H, Tabrizi JS and Azami-Aghdash S., Barriers to evidence-based medicine: a systematic review. J. of Evalu in Clinical Prac (2014) [doi:10.1111/jep.12222].

[18] Zwolsman SE, Pas E te, Hooft L, Wieringa-de Waard M and van Dijk N., Barriers to GPs' use of evidence-based medicine: a systematic review $\mathrm{Br} J$ Gen Pract (2012) [DOI: 10.3399/bjgp12X652382]

[19] Grimshaw J., Shirran L., Thomas R, Mowatt G, Fraser C, Bero L, et al., Changing provider behavior: an overview of systematic reviews of interventions. Med Care 39(8 Suppl 2):II2-45 (2001).

[20] Grimshaw J., Eccles MP., Walker AE. and Thomas RE., Changing physicians' behavior: what works and thoughts on getting more things to work. J Contin Educ Health Prof 22(4):237-43 (2002).

[21] Belizan M, Meier A, Althabe F, Codazzi A, Colomar M, Buekens P, et al., Facilitators and barriers to adoption of evidence-based perinatal care in Latin American hospitals: a qualitative study. Health Educ Res 22:839-53 (2007).

[22] Okwundu CI., Knowledge of Evidence-Based Medicine (EBM) terminologies and use of EBM resources at the University of Ilorin Teaching Hospital, Nigeria. Abstracts of the Joint Cochrane and Campbell Colloquium. Oct 18-22, 2010, Keystone, Colorado. CDSRs, (Suppl 2010): Art No: CD000002. DOI:10.1002/14651858.CD000002.

[23] Gudala K., Bansal D., and Kanukula R., Barriers and methods to improve the use of Cochrane systematic reviews as a primary source of evidence in developing countries. In: EvidenceInformed Public Health: Opportunities and Challenges. Abstracts of the $22^{\text {nd }}$ Cochrane Colloquium; 2014 21-26 Sep; Hyderabad, India. John Wiley \& Sons.(2014)

[24] Ajuwon GA., Use of the Internet for health information by physicians for patient care in a teaching hospital in Ibadan, Nigeria. Biomed Digit Libr 3:12 (2006). 
Relevance and Barriers to Evidence-Based Practice in Zambian Defense Force Medical Services: A Qualitative Analysis of Roundtable Group Discussion by Military Health Personnel

[25] Abdulwadud O, Young T, Hailu K, Rangwala A, Midekssa M, Volmink J and Garner P, Evidence based practice workshop for senior managers in Ethiopia. Abstracts of the 19th Cochrane Colloquium. Oct 19-22, 2011, Madrid, Spain. CDSRs, (Suppl 2011): Art No.: CD000003.

[26] Oliver J and Young T., What can The Cochrane Collaboration do to support people living in developing countries? A survey. In: Corroboree. Abstracts of the 13th Cochrane Colloquium 2005 22-26 Oct; Melbourne, Australia (2005)

[27] Abdulwadud OA., Raising awareness about the Cochrane Collaboration and South African Cochrane Centre in Ethiopia. Abstracts of the Joint Cochrane and Campbell Colloquium. October 18-22, 2010, Keystone, Colorado. CDSRs, (Suppl 2010): Art No: CD000002. DOI:10.1002/14651858.CD000002.

[28] Nwagwu W., Levels of consciousness and awareness about evidence-based medicine among consultants in tertiary health care institutions in Nigeria. Health Info Libr J 25(4):278-87 (2008).

[29] Sylla AH, Robinson ET, Raney L and Seck K., Qualitative study of health information needs, flow, and use in Senegal. J Health Commun 17 Suppl 2:46-63 (2012) [DOI: $10.1080 / 10810730.2012 .666624]$.

[30] The Zambian national regulatory authority.Accessed 10 February 2016 [https://en.wikipedia.org/wiki/Clinical_officer\#Zambia]

[31] OpenCode 4.03 ( ) ITS and Division of Epidemiology and Global Health, Department of Public Health and Clinical Medicine. Umeå University, Sweden 2011.

[32] Agarwal R., Kalita J., and Misra UK., Barriers to evidence based medicine practice in South Asia and possible solutions. Neurol Asia 13:87-94 (2008).

[33] Lindner JR., Understanding Employee Motivation. The journal of Extension 36(3) (1998). [http://www.joe.org/joe/1998june/index.php]

[34] Rohwer A and Young T., Building capacity in EBHC: What is happening in the African region? In: Abstracts of the 19th Cochrane Colloquium; 2011 19-22 Oct; Madrid, Spain. John Wiley \& Sons (2011)

[35] Martis R, Ho JJ. and Crowther CA. for The SEA-ORCHID Study Group. Survey of knowledge and perception on the access to evidence-based practice and clinical practice change among maternal and infant health practitioners in South East Asia. BMC Preg and Childbirth 8:34 (2008) [doi:10.1186/1471-2393-8-34].

[36] Dawes M., Summerskill W., Glasziou P., Cartabellotta A., Martin J., Hopayian K, et al., Sicily statement on evidence-based practice. BMC Med Educ 5:1 (2005) [doi:10.1186/1472-6920-5-1]

[37] Kotur PF., Introduction of evidence-based medicine in undergraduate medical curriculum for development of professional competencies in medical students. Curr Opin Anaesthesiol 25:719$23(2012)$ 